\title{
Endobronchial argon plasma coagulation for the palliation of recurrent tracheobronchial adenoid cystic carcinoma
}

\author{
Masanori Yasuo MD, PhD*, Tsuyoshi Tanabe MD *, Kenji Tsushima MD, $\mathrm{PhD}$ *, \\ Yoshimichi Komatsu MD*, Hiroshi Yamamoto MD, PhD*, PhD, \\ Masayuki Hanaoka MD, PhD*, Tomonobu Koizumi MD, $\mathrm{PhD}$ *, \\ Keishi Kubo MD, $\mathrm{PhD}$ *, Toshihide Wakamatsu MD**, Yoshiki Hirose MD, PhD \\ ***, and Yoshitaka Yamazaki MD, $\mathrm{PhD}$ **** \\ * First Department of Internal Medicine, Shinshu University School of Medicine, \\ ** Department of Respiratory Medicine, Nagano Red-Cross Hospital, \\ ***Department of Internal Medicine, Ina Central Hospital \\ ****Department of Endoscopy, Shinshu University School of Medicine, \\ Nagano, JAPAN
}

Short title: Endobronchial argon plasma coagulation

Correspondence: Masanori Yasuo

$1^{\text {st }}$ Department of Internal Medicine

Shinshu University School of Medicine

3-1-1 Asahi, Matsumoto, Japan

Telephone: +81 26337 2631, Fax: +81 263363722

E-mail address: yasumasa@hsp.md.shinshu-u.ac.jp

Words: 1498 words 


\begin{abstract}
The standard treatment of adenoid cystic carcinoma (ACC) of the airway is surgery if possible, since survival rate is better than other treatments including radiotherapy. Although ACC shows frequent recurrence during the long term follow up unless there has been a complete resection (negative surgical margin), no further treatments are recommended. This report describes how argon plasma coagulation using flexible bronchoscopy has been successfully employed in the treatment of ACC after conventional therapy in one case of recurrence after surgery and two cases of inoperable patients. All of the patients are alive and healthy more than six years after diagnosis.
\end{abstract}

Key words: palliative therapy; flexible bronchoscopy; argon plasma coagulation; endobronchial therapy; electrocoagulation 


\section{Introduction}

Adenoid cystic carcinoma (ACC) of the airway is a rare primary tumor that occurs in all adult age groups and is frequently slow to progress and late to metastasize or recur locally [1]. Although tumors of the trachea are much less common than those found in the lungs, squamous cell carcinoma and ACC are the most frequent histological types [2]. These lesions are usually resistant to nonsurgical therapy especially to chemotherapy. Many cases are open to a segmental resection of the airway with removal of all gross disease and reconstruction by primary anastomosis [1]. Gaissert et al. has reported relatively good results in 101 resected lesions in a total of 135 patients with ACC [2]. However, not all patients could undergo surgery because of a delay in diagnosis, the propensity of the tumor to spread submucosally and along perineural bundles and the difficulty of resection due to the length limit (up to $6 \mathrm{~cm}$ ) [1-3]. Furthermore, a high rate of recurrence and incomplete resection has been reported $[1,2]$. Palliative therapy for airway management was required for those patients. The use of lasers for treating tracheobronchial ACC has been reported [3, 4], however standard the palliative therapeutic strategy is not presented due to the low incidence of this disease.

Argon plasma coagulation (APC) has recently been widely used in tracheobronchial disease $[5,6]$ because it allows a rapid coagulation with minimal manipulation and mechanical trauma to the target tissue [6]. A previous study reported the safety efficacy of APC for the management of airway stenosis [7]. APC was also used for the management of recurrent three cases of ACC after several treatments, including tracheal reconstruction, radiation and brachytherapy etc. This report will discuss the safety and usefulness of this therapy for palliative airway management.

\section{Case Report}

Table 1 shows clinical details and follow-up of our cases.

Case 1

A 48-year-old male was admitted to the hospital for hemosputum in January 1999. Fiberoptic bronchoscopy revealed a lower tracheal mass which was diagnosed as ACC by transbronchial biopsy. The patients underwent a tracheal resection which was followed by postoperative radiation because of incomplete resection. In 2001, tumor recurrence at the left second spur was verified by surveillance bronchoscopy. Systemic chemotherapy (cisplatin and docetaxel), external radiation (30.6Gy) and brachytherapy (13Gy) were performed from March 2001 to January 2002. However, 
the lesion at the left second spur still remained. APC was applied from February 2002. The equipment for APC consists of an APC probe, an argon-gas source, and a high-frequency surgical unit (APC 300, ERBOTOM ICC; Erbe Elektromedizin; Tuebingen, Germany). To deliver the gas, a flexible-monopolar Teflon tube was used which had a 1.5-mm diameter and a 150-cm length. This tube was put into the working channel of a flexible bronchoscope. Several short bursts of APC were administered. The APC procedure was performed about every two months (total 34times). No adverse effect has seen and the patient is now working full time at his company.

\section{Case 2}

A 43-year-old female was admitted to the hospital for chronic cough in October 2001. Central airway stenosis was implied by a pulmonary function test. Fiberoptic bronchoscopy revealed a tracheal mass at the bottom of the trachea which was diagnosed as ACC. Tracheal reconstruction was determined to be impossible because of severe invasion of the carina. A Y-shaped Dumon stent was implanted one month after the diagnosis. She was then treated with 60Gy of external radiation therapy. The stent was removed on July 2003. In June 2004, tumor recurrence was observed in the carina. APC was applied beginning from July 2004. Several short bursts of APC were administered and the devitalized tissue was then mechanically removed with grasping forceps. The APC procedure was performed every two to three months (total 18 times). The patient complained of slight chest discomfort when the bursts were performed. No other adverse effects were seen and she is now working full time as an office worker.

\section{Case 3}

A 66-year-old female was admitted to the hospital for further palliative therapy of recurrent ACC. She was diagnosed with ACC at the trachea and surgical resection was determined to bed impossible in another hospital because of considerable spread of the disease in August 2000. She received external thoracic radiation and tumor reduction with a potassium-titanyl-phosphate laser with conscious intubation several times. In 2001, brachytherapy was performed at the trachea. In 2003, a metastatic lesion in the left upper lobe of her lung was detected and she underwent a partial resection of the left upper lobe. In 2005, ACC recurrence was found in the trachea. Then she again received several rounds of potassium-titanyl-phosphate laser therapy. However tumor recurrence was not 
controlled in the trachea and a new lesion appeared on the left upper bronchus. She requested another palliative therapy without intubation, and she was referred to this department in November 2006. APC was administered for these lesions. Several short bursts of APC were administered at the two sites of recurrence, and the devitalized tissue was then mechanically removed with grasping forceps (Figure 1A, B). The APC procedure was performed every one to two months (total 7times). No adverse effects have occurred and she is now able to lead a normal life as a housewife. 


\section{Discussion}

This study describes the use of APC against ACC after standard therapies have been unsuccessful. The APC provided keep tracheobronchial patency in patients with recurrent ACC after either reconstructive surgery or when surgery was considered impossible.

Longer survival has been reported in patients that underwent a resection for ACC than those who did not [2]. The largest study of ACC (135 cases) indicated a significant survival benefit associated with complete resection and negative airway margins [2]. The radiosensitivity of cervico-cranial ACC has been demonstrated [8]. In addition, radiotherapy of tracheobronchial ACC is also recommended especially when a positive margin is observed after surgery and for palliative therapy [3]. However, recurrence will inevitably occur after these established therapies. Considering the pathophysiological characteristics of this disease, further palliative therapy for maintaining airway patency must be applied. Nd-YAG laser photoresection is the most common strategy for palliative treatment in unresected and recurrent ACC $[4,9]$. Patients who received laser therapy experience immediate symptomatic relief and relatively good results for palliative therapy [2, 4, 9]. However, Nd-YAG laser photoresection usually requires a rigid bronchoscope and general anesthesia, and fatal adverse effects such as hemorrhage have been reported during and after this therapy [9]. For these reasons, repetitive treatment for keeping airway patency seems somewhat difficult in most therapeutic units.

Recently, APC has been used in the treatment of benign or malignant lesions in the airway [5-7]. It has an excellent safety profile, is convenient and simple to use, and is relatively inexpensive. These characteristics make it suitable for therapeutic applications through a flexible bronchoscope [6]. Morice et al. [6] showed that APC devitalizes tissue gradually by producing temperatures that coagulate and desiccate tissue. This can result in a homogenous but limited depth of penetration (approximately $3 \mathrm{~mm}$ ). As a result, APC offers uniform coagulation and good protection against airway perforation and hemorrhage [6]. No adverse events occurred during the 59 treatment courses in the three cases described in this report. In addition, no patients required oxygen supplementation during the therapeutic periods in this series.

This report documents the use of APC against ACC in only three cases, therefore, there is no available data about the prognosis. However, all these cases have maintained good performance status for more than 6 years. Because the largest series addressing ACC treatment [2] reported that the overall 5- and 10- year 
survival in resectable and unresectable ACC were $52 \%$ and $33 \%$, this palliative airway management strategy appears to be effective.

In summary, repetitive endobronchial dilatation was performed using APC in three cases of ACC after standard therapies had been insufficient. APC was able to provide a further palliative therapeutic choice for maintaining airway patency in patients with recurrent tracheobronchial ACC. 


\section{References}

1. Maziak DE, Todd TR, Keshavjee SH et al. Adenoid cystic carcinoma of the airway: thirty-two-year experience. J Thorac Cardiovasc Surg 1996; 112: 1522-32.

2. Gaissert HA, Grillo HC, Shadmehr MB et al. Long-term survival after resection of primary adenoid cystic and squamous cell carcinoma of the trachea and carina. Ann Thorac Surg 2004; 78: 1889-97.

3. Clough A, Clarke P. Adenoid cystic carcinoma of the trachea: a long-term problem. ANZ J Surg. 2006; 76: 751-3.

4. Albers E, Lawrie T, Harrell JH et al. Tracheobronchial adenoid cystic carcinoma: a clinicopathologic study of 14 cases. Chest. 2004; 125: 1160-5.

5. Tremblay A, Marquette $\mathrm{CH}$. Endobronchial electrocautery and argon plasma coagulation: a practical approach. Can Respir J. 2004; 11: 305-10.

6. Morice RC, Ece T, Ece F et al. Endobronchial argon plasma coagulation for treatment of hemoptysis and neoplastic airway obstruction. Chest. 2001; 119: 781-7.

7. Yasuo M, Tanabe T, Tsushima K et al. Endobronchial argon plasma coagulation for the management of post-intubation tracheal stenosis. Respirology. 2006; 11: 659-662.

8. Khan AJ, DiGiovanna MP, Ross DA et al. Adenoid cystic carcinoma: a retrospective clinical review. Int J Cancer. 2001; 96:149-58.

9. Díaz-Jiménez JP, Canela-Cardona M, Maestre-Alcacer J. Nd:YAG laser photoresection of low-grade malignant tumors of the tracheobronchial tree. Chest. 1990; 97: 920-2. 


\section{Figure legends}

Figure 1 A; Bronchoscopic findings of recurrent ACC of the trachea. The intraluminal space was markedly reduced. B; One month after the second APC treatment, the site of stenosis was well dilated. 